\title{
20
}

\section{ANÁLISIS DE LOS SISTEMAS DE GESTIÓN DEL CONOCIMIENTO EN LA EMPACADORA GRUPO GRANMAR: DIVISIÓN LABORATORIO DE LARVAS SEMACUA S.A DE LA PROVINCIA DE SANTA ELENA - ECUADOR.}

Mónica Jaramillo, Washington Torres. 


\title{
ANÁLISIS DE LOS SISTEMAS DE GESTIÓN DEL CONOCIMIENTO EN LA EMPACADORA GRUPO GRANMAR: DIVISIÓN LABORATORIO DE LARVAS SEMACUA S.A DE LA PROVINCIA DE SANTA ELENA - ECUADOR
}

\section{ANALYSIS OF KNOWLEDGE MANAGEMENT SYSTEMS IN THE PACKING GROUP GRANMAR: LABORATORY DIVISION LARVAS SEMACUA S.A PROVINCE OF SANTA ELENA - ECUADOR}

\author{
Mónica Jaramillo ${ }^{1}$, Washington Torres ${ }^{2}$ \\ Universidad Estatal Península de Santa Elena (UPSE) \\ Campus La Libertad, Vía Principal Santa Elena - La Libertad \\ La Libertad-Ecuador \\ monyjinfante@gmail.com ${ }^{1}$, wtorres@upse.edu.ec ${ }^{2}$
}

\section{Resumen}

La gestión de los activos intangibles que generan valor para la organización son aquellos que tienen que ver con los procesos relacionados con la transmisión del conocimiento, por este motivo se diseña un modelo de gestión del conocimiento, con el uso de herramientas TIC, para agilizar el proceso y mejorar el uso de los mecanismos de acceso y búsqueda de información, puesto que los que funcionan actualmente no garantizan una correcta transferencia de información ni el aprendizaje adecuado, la creciente importancia del conocimiento como un nuevo factor de producción hace que el desarrollo de tecnologías, metodologías y estrategias se convierta en una de las principales prioridades de las organizaciones en la sociedad del conocimiento. Se conoce que el mercado es cada vez más competitivo, lo que demanda mayor innovación en los productos, por ello, el conocimiento debe desarrollarse y ser asimilado cada vez con mayor rapidez, lo que se logra al tener un proceso automatizado que cumpla con políticas de seguridad para el tratamiento de la información.

El modelo de gestión está orientado a las personas que aplican tecnología enfocada al intercambio de conocimientos, debido a que las empresas aprovechan al máximo sus conocimientos, no repiten tareas ni pierden tiempo en realizarlas para compartir el conocimiento en la organización.

Palabras Claves: gestión del conocimiento, TIC

\section{SUMMARY}

The management of intangible assets that generate value for the organization are those that have to do with the processes related to the transmission of knowledge, for this reason a model of knowledge management is designed, with the use of ICT tools to streamline the process and improve the use of the access mechanisms and finding information, since those operating not currently guarantee a correct transfer of information or adequate learning, growing importance as a new production factor has turned development of technologies, methodologies and strategies to become one of the main priorities of organizations in the knowledge society. It is known that the market is increasingly competitive, which demands greater innovation in products, therefore, knowledge must be developed and assimilated more and more quickly, which is achieved by having an automated process that meets policies security for information processing. The management model is aimed at people who apply technology focused knowledge sharing, because the companies take full advantage of their knowledge, repeat not waste time tasks or perform them to share knowledge in the organization.

Keywords: knowledge management, ICT 


\section{Introducción}

Los nuevos modelos de gestión del conocimiento no excluyen ninguna de las prácticas realizadas con anterioridad, sino que se añade una nueva en un aspecto muy humano de la organización como es la aplicación del saber.

La tecnología aporta la inteligencia a los sistemas que las personas tienen que manejar, pues las situaciones que hay que resolver en un entorno tecnológico son mucho más complejas cualitativa y cuantitativamente, y sólo a través de sistemas complejos y en régimen de colaboración entre muchas personas es posible resolver los nuevos problemas. En cualquier caso, ya no basta con saber los fundamentos de las cosas ni manejar con habilidad los instrumentos elementales. Cada uno depende en su actividad profesional de sistemas complejos y tiene que ser capaz de dominarlos y sacarles el máximo rendimiento.

\subsection{Problemas que se derivan en la información esparcida}

La ausencia de datos integrados y de herramientas TIC para su explotación, sugiere que los portales colaborativos, deberían tener la capacidad integrar diversas tecnologías, permitir la estandarización tecnológica, reducir gastos y a su vez optimizar los procesos. Desde el punto de vista de la gestión de capacidades tecnológicas, refiere a la nueva empresa inteligente la cual gestiona el conocimiento, no es lo mismo datos que información, debido a que este se basa en identificar, estructurar y utilizar la información para alcanzar un resultado, más aún en las empresas actuales que dan cambios rápidos, por lo que deben encontrar nuevas formas de utilizar el conocimiento que disponen y donde entra la gestión del conocimiento.

\subsection{Importancia de los modelos de Gestión del conocimiento}

La creciente importancia del conocimiento como un nuevo factor de producción hace que el desarrollo de tecnologías, metodologías y estrategias hace que se convierta en una de las principales prioridades de las organizaciones en la sociedad del conocimiento. Se conoce que el mercado es cada vez más competitivo, lo que demanda mayor innovación en los productos. Debido a esto, el conocimiento debe desarrollarse y ser asimilado cada vez con mayor rapidez, lo que se logra al tener un proceso automatizado que cumpla con políticas de seguridad para el tratamiento de la información.

\section{Métodos y Técnicas}

El método teórico que se utilizará es el hipotético - deductivo, debido a que se realizará una investigación y observación de la realidad obteniendo así un análisis para determinar la hipótesis para la solución de la inexistencia de datos integrados y de herramientas TIC.(Newman, Gladys, \& Newman, 2007).

Este método teórico contribuirá mucho con el proceso de recolección de información sobre el problema existente dentro de la Empacadora Grupo GRANMAR: División Laboratorio camaronero SEMACUA S.A de la Provincia de Santa Elena, ubicado en el cantón Salinas, debido a que los datos que sean recolectados sobre la pérdida de tiempo en la generación de información originada por la falta de datos integrados y de herramientas TIC, ya que se realizará un análisis y desarrollar un modelo que de soporte a la transferencia del conocimiento.

La encuesta es un método complementario que se realizará al personal que trabaja en el Laboratorio de larvas SEMACUA S.A., con la finalidad de obtener información de las personas que viven la realidad del problema ya que dicha recopilación de datos también contribuirá para la solución al problema y así mismo será un complemento confirmado luego de haber realizado una observación de los procesos que se realizan en la situación actual, es por este motivo que al hacerla encuesta nos permitirá ejecutar un estudio estadístico de los resultados obtenidos para un posterior análisis de los requerimientos específicos que tenga la empresa (Chiner, 2011).

\subsection{La Información y su tratamiento estadístico}

El diseño de la investigación se basará en análisis comparativos que permitan recolectar información cuantitativa y cualitativa, debido a que se requiere analizar cada proceso que se realice específicamente al control y funcionamiento del modelo de gestión.

La información que se logra obtener se tabularán y resumirán en gráficos estadísticos, los cuales se irán desarrollando de forma automática, para luego ser presentados de forma escrita, para lo cual también agregaremos un gráfico en la cual se determinará escalas de medición referente a la población estudiada.

Mediante la evaluación de las herramientas más reconocidas en el mercado, identificando los servicios y los recursos tecnológicos requeridos para la implementación de portales colaborativos 
de trabajo. Va a permitir luego del modelo la implementación de un producto final, puesto que se identifican los factores técnicos los cuales deberán tener mayor incidencia en la creación de este modelo.

El estudio realizado generará muchas facilidades, ahorro de tiempo y gastos, debido a que el uso de herramientas TIC dentro de una organización no solo contribuye con el desarrollo a nivel de tecnología en una organización, sino que también facilita y disminuye el tiempo laboral, ya que la tecnología está dirigida a la visión de brindar efectividad y eficacia, de esta manera se pueden implementar este tipo de tecnologías para crecer tanto organizacionalmente como a nivel de conocimientos del personal.

En este caso se hará uso de la tecnología para brindar facilidad de acceso a la información que actualmente se encuentra dispersa, lo que demanda tiempo de búsqueda y gastos económicos, al lograr integrar dicha información no solo se evita estos tipos de inconvenientes, sino que también facilita al personal acceder a la información en el momento que se requiera y desde el lugar en que se encuentre con mayor rapidez, para ello se debe capacitar al personal para que le den el correcto uso a esta herramienta de trabajo.

Debido a que contribuirá con la facilidad de acceso a la información, se debe realizar un mantenimiento de datos, lo que implica actualización, edición y eliminación de información, brindando así datos valiosos que sea de mucha utilidad para que el personal no tenga inconvenientes, dicha información puede ser mejorada de acuerdo a como amerite, por ende la organización dispondría de información actualizada frecuentemente, para esto se debería designar a un administrador responsable de los datos brindados. Así mismo mejoraría el ambiente laboral, ya que aumentará la comunicación en el personal de la organización, ya que mejorarán sus conocimientos tanto a nivel de la información organizacional como a nivel tecnológico, contando de esta manera con un personal debidamente capacitado y un ambiente de trabajo productivo que contribuya con el crecimiento de la empresa.

Para la implementación de dicho sistema se ha tomado en cuenta aspectos indispensable que implica: el modelo, diseño y uso aplicaciones que faciliten el trabajo, uno de los aspectos importantes es que la empresa contaría con una base de datos que almacene la información que sea necesaria, facilitando el acceso y búsqueda de datos, así como también la implementación de otro tipo de herramientas que ayude con la solución a dicho problema, por ciertos aspectos como la reutilización de la información.
Actualización de información que vaya acorde a los conocimientos adquiridos, logrando así una mayor organización del manejo de información y la aceptabilidad del personal a los nuevos procesos. Se requiere de capacitaciones tanto para el personal que lo administre como para el personal que haga uso del sistema, por ende se deben implementar políticas con respecto al manejo de información.

2.2. Análisis comparativo, evolución, tendencia y perspectivas

La Empacadora Grupo GRANMAR: División Laboratorio camaronero SEMACUA S.A de la Provincia de Santa Elena, ubicado en el cantón Salinas, no cuenta con el uso de las herramientas TIC lo que ocasiona pérdidas de tiempo en la gestión de la información, en base a la encuesta realizada al personal del laboratorio indica que es necesario buscar estrategias que mejoren la factibilidad y agiliten dicho proceso, adaptarse al manejo y uso de las herramientas TIC.

Se considera que al implementar el uso de la herramientas TIC en el proceso de gestión de información se evitaran pérdidas de tiempo que indica que será de mucho beneficio para el personal ya que los procesos manuales serian reemplazados por el uso de la tecnología y lo que antes le tomaba de unos 20 a 30 minutos puede disminuirse a unos 10 minutos, así como también se evitaría el riesgo que existe al manipular información de manera manual. El hecho de implementar herramientas TIC en el laboratorio beneficiará no solo en evitar la pérdida de tiempo sino que también contribuirá con el desarrollo tecnológica del laboratorio formando así red no solo física sino también de información, así como también incentivara a seguir creciendo tecnológicamente y no solo físicamente.

La perspectiva de herramientas TIC es que colocar al Laboratorio camaronero SEMACUA S.A de la Provincia de Santa Elena en un ambiente tecnológico que este en la capacidad de realizar procesos eficaces y a su vez implementar nuevo procesos con la ayuda de los sistemas informáticos y herramientas TIC, de esta manera se estaría contribuyendo con la evolución tecnológica ya que de esta manera no solo estará enfocado en la competitividad de mercado sino también en la competitividad de transferencia de conocimiento en medio de comunicación virtuales. Para diseñar el modelo se reorganizaron los procedimientos que eran realizados de forma manual, esto nos permitió modelar un sistema de gestión del conocimiento que va a permitir un dinamismo en el flujo de la información. 


\section{Resultados}

\subsection{Tiempos de respuesta en la generación de} la información

El mecanismo de transferencia de información actualmente es regular, por lo que es notable que el nivel de satisfacción debe mejorar, motivo por el cual se ha considerado buscar estrategias para mejorar dicho proceso.

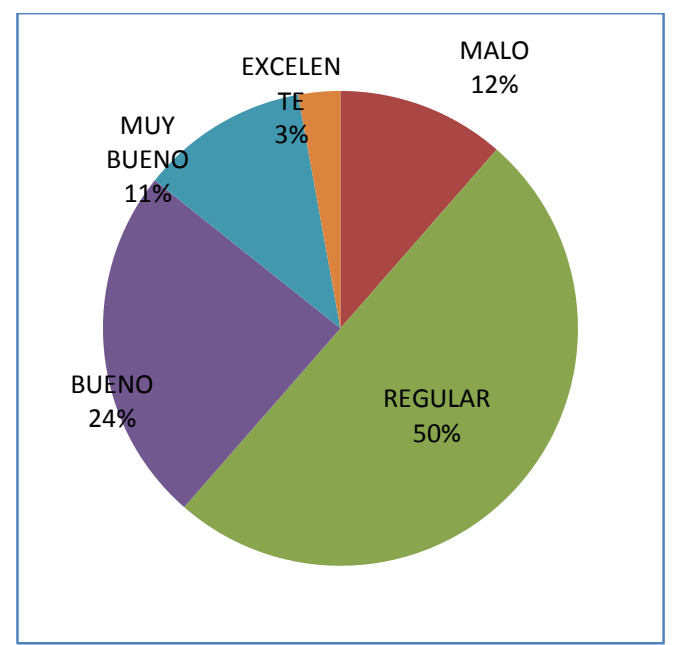

Figura No 1 Tiempos de respuesta en generar la información

\subsection{Motores de búsqueda para acceder a la} información de la organización

El rango de usuarios que acceden a los sistemas de información es de 6 a 10, con la finalidad de administrar datos, cabe recalcar que dichos usuarios no ha sido capacitados, pero gracias a la interfaz que brinda el sistema trabajan de manera empírica, mientras que existe también un porcentaje considerable de los jefes departamentales indicando que han sido capacitados para darle el uso correcto a dicho sistema de información.

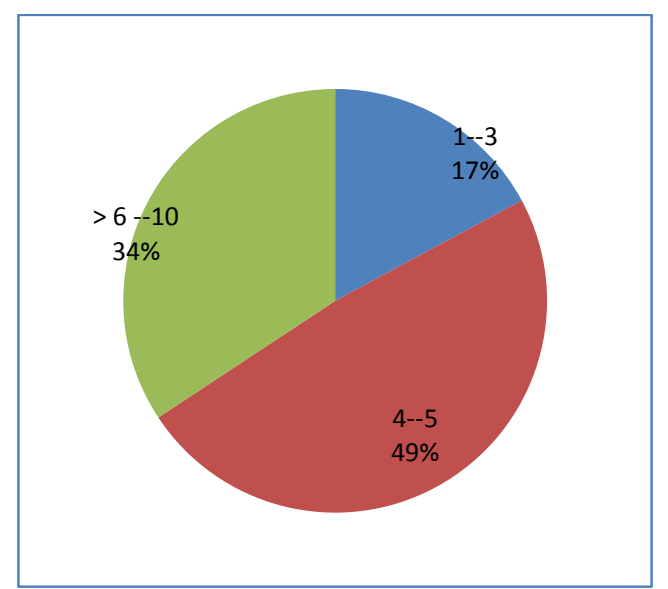

Figura No 2 Frecuencia de motores de búsqueda para acceder a la información

3.3. Nivel de conocimiento sobre las TIC que poseen los usuarios de la organización

Se deduce que el 14\% de los usuarios tienen mucho conocimiento de las TIC, mientras que un alto porcentaje indican que no aplican el acceso a metodologías de transferencia del conocimiento, lo cual indica que existe una pérdida de tiempo considerable en este proceso, el mismo que debe ser mejorado aplicando estrategias tales como la implementación del uso de las herramientas TIC, lo que va a permitir agilizar el proceso.

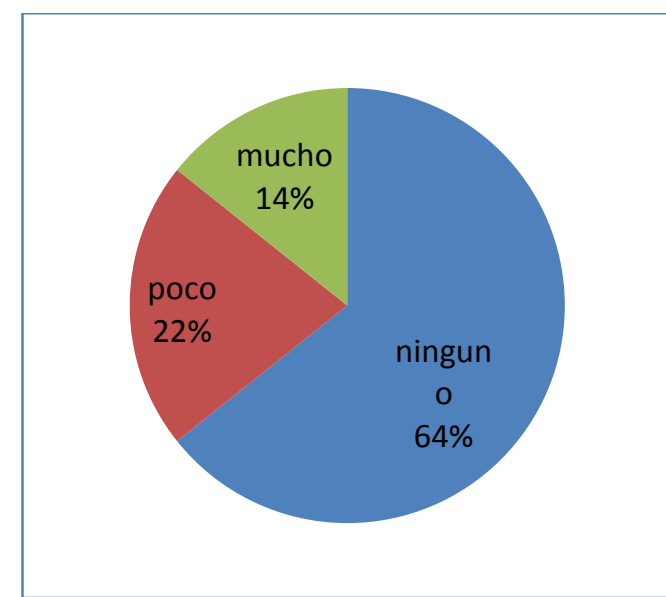

Figura No 3 Gráfico estadístico de Metodologías o mecanismos de transferencia

3.4. Metodologías o mecanismos de transferencia de conocimiento aplican la organización

los resultados que se obtuvo del registro de funcionalidades notamos que un $90 \%$ de las herramientas evaluadas incluyen foros de discusión electrónica, el 100\% de los productos incluye la herramienta del correo electrónico como parte de sus servicios, el 100\% incluye herramientas de difusión de información y administración de contenidos para controlar los 
procesos de la empresa, el $74 \%$ de los productos incluyen como herramienta los mensajes instantáneos o chats y finalmente, se detecta como algo muy significativo que sólo el $26 \%$ de los productos incluyen pequeñas herramientas de administración del conocimiento con la finalidad de potenciar la transmisión del conocimiento en su capital intelectual.

\subsection{Discusión}

Según Valhondo (2003) El conocimiento es un recurso que está convirtiéndose en una materia con un enorme potencial para cambiar el mundo debido a los avances de las nuevas tecnologías de la información. En el entorno económico en el que nos encontramos, el conocimiento es un elemento esencial para la economía de la información e implica la creación de herramientas que permitan una gestión correcta de este conocimiento.

Aunque Goñino establece en la conceptuación del modelo la forma de obtener estos resultados, es interesante la integración que realiza de las etapas que define para que el modelo resulte realmente dinámico en su implementación.

El estudio realizado generará muchas facilidades, ahorro de tiempo y gastos, debido a que el uso de herramientas TIC dentro de una organización no solo contribuye con el desarrollo a nivel de tecnología en una organización, sino que también facilita y disminuye el tiempo laboral, ya que la tecnología está dirigida a la visión de brindar efectividad y eficacia, de esta manera se pueden implementar este tipo de tecnologías para crecer tanto organizacionalmente como a nivel de conocimientos del personal.

En este caso se hará uso de la tecnología para brindar facilidad de acceso a la información que actualmente se encuentra dispersa, lo que demanda tiempo de búsqueda y gastos económicos, al lograr integrar dicha información no solo se evita estos tipos de inconvenientes, sino que también facilita al personal acceder a la información en el momento que se requiera y desde el lugar en que se encuentre con mayor rapidez, para ello se debe capacitar al personal para que le den el correcto uso a esta herramienta de trabajo.

\section{Conclusiones}

Después del estudio realizado se considera que se debe administrar eficientemente los conocimientos ingresados por los usuarios, para de esta manera lograr procesos eficientes, efectivos y por ende disminuir gastos.

Al implementar la plataforma web hace que la información esté disponible, pudiendo ser acceder desde cualquier parte y en el momento que se requiera, evitando pérdidas de tiempo en búsquedas de información.

La estrategia de hacer uso de la estructura del árbol facilitará la creación, organización y búsqueda de los conocimientos en el sistema manteniendo así un proceso jerárquico.

$\mathrm{Al}$ contar con una herramienta capaz de preservar en el tiempo los conocimientos que han sido calificados como valiosos, hace que se cumpla la expectativa de hacer uso de las herramientas TIC para la administración de información.

La facilidad de acceso a los conocimientos estarán sin restricción, lo que permite que puedan ser revisados por todos los usuarios sin limitación alguna, ya que los mismos puede ser útiles en casos que lo amerite y los mismos a su vez pueden contribuir para ser mejorados.

La disponibilidad de promover la reutilización de los conocimientos aprovechando la experiencia de otros para ahorrar tiempo, lo cual contribuirá para agilización de procesos que ameriten de los mismos conocimientos para ser ejecutados.

Los cambios en la tecnología también implican tener actualizado al personal dispuesto a prepararse para afrontar el desafío que impone el mercado actual, debido a ellos cumplen sus tareas por intermedio de la misma.

\section{Agradecimiento}

Mi más sincero agradecimiento a la Ing. Lilia Valencia Cruzaty, Rectora de la Universidad Estatal Península de Santa Elena por su gestión y el aporte a la comunidad universitaria en difusión de artículos formativos de mucha importancia; y a quienes colaboraron para que este trabajo se diera de la mejor forma.

\section{Bibliografía}

1. Bolaños Rodríguez, E. (2012). Muestra y Muestreo.

2. Chiner, E. (2011). Investigación Descriptiva Mediante Encuestas, 1-18. Retrieved from http://rua.ua.es/dspace/bitstream/10045 /19380/34/Tema 8-Encuestas.pdf

3. Estad, L., Producci, S. D. E. L. A., Ch, H. L., \& Herrera, G. Z. (n.d.). No Title.

4. Gesti, L. (n.d.). LA GESTIÓN DEL CONOCIMIENTO.

5. Newman, D., Gladys, L., \& Newman, D. (2007). Experimentales Y Sociales.

6. PUENTE, M. D. LA. (2010). GESTION DEL CONOCIMIENTO Y MINERIA DE DATOS, DOCUMENTOS.

7. Rodríguez-Gómez, D. (2006). Modelos para la creación y gestión del conocimiento: una aproximación 
teórica.Educar, 37, 25-39. Retrieved

from

http://www.raco.cat/index.php/Educar/ article/viewArticle/58019/0

8. Tarí-Guilló, J., \& Garcìa-Fernández, M. (2009). Dimensiones de la gestión del conocimiento y de la gestion de la calidad: una revision de la literatura. Investigaciones Europeas de Dirección Y Economía de La Empresa, 15(3), 135-148.

9. Valhondo, D. (2003). Gestión del conocimiento: del mito a la realidad.

10. Von Krogh, G. (1998). Care in Knowledge Creation. California Management Review, 40(3), 133-153. http://doi.org/10.2307/41165947. 